\title{
EPILOGUE
}

\section{From Introspections, Brain Scans, and Memory Tests to the Role of Social Context: Advancing Research on Interaction and Learning}

\author{
Alison Mackey \\ Georgetown University
}

The goal of this epilogue is to use the methodological contributions of the studies presented in this special issue as a starting point for suggestions about methodology in conducting future interaction research. As is the case in most developing fields, interaction research develops methods internally as it continually borrows and extends techniques used in other disciplines and revitalizes older techniques by adding new or different angles unique to interaction. Interaction researchers have also begun to forge relationships in new areas (e.g., by working with psychologists and developing working memory [WM] tests). This sort of cooperation is an important step in the drive to uncover more information about the relationship between interaction and learning. As several contributors to this special issue have noted, the most recent advances in methodology have been driven by questions about how interaction works (as opposed to whether it works). In turn, some of the methodological innovations discussed here will also ultimately allow new questions to be asked. Indeed, the relationship between questions (i.e., suggestions about what needs to be investigated next) and methods (i.e., plans for how to carry out such investigations) is particularly close in interaction research, which is a relatively new but vibrant and quickly developing area. Consequently, this epilogue considers both methods and questions conjointly, beginning with a

I am grateful to Helen Carpenter, Akiko Fujii, Susan M. Gass, Mika Hama, Jennifer Leeman, Kim McDonough, and Jenefer Philp for various kinds of help, including discussions of ideas and feedback, in relation to an earlier version of this paper, and to Amanda Edmonds and the other SSLA editorial staff members for their outstanding work and assistance with this Epilogue and the entire special issue. Any errors are, of course, my own.

Address correspondence to: Alison Mackey, Department of Linguistics, 460 Intercultural Center, Georgetown University, Washington, DC 20057-1051; e-mail: mackeya@georgetown.edu. 
discussion of methodological issues in the most recent theorizing about the interaction hypothesis.

\section{RECENT ACCOUNTS OF THE INTERACTION HYPOTHESIS AND RESEARCH METHODOLOGY}

The original and seminal claims about interaction and learning were made by Long and, fittingly, he has recently pointed the way in terms of considerations in the design of interaction research (Long, in press). He argued that definitions of terms should be clear and, where possible, standardized across studies. Many terms, such as recasts, are increasingly considered in the literature as somewhat elastic, with researchers operationalizing them in terms of more and less explicit, simple versus complex, long versus short, declarative versus interrogative, and so forth (see also Loewen \& Philp, in press). Long also suggested that researchers need to pay attention to the differences between acquisition and deployment of forms in interaction research (a point elaborated on in what follows) because these differences also involve the nature of knowledge and affect the design, use, and interpretation of outcome measures and tests. Moreover, the importance of these differences holds for other types of SLA research as well. Long noted that research attention also needs to be directed to the importance of timing in the design of studies concerned with the provision and use of feedback (see also Lightbown, 1998; Pica, 1994). He pointed to the need for work in different modalities (e.g., in L2 writing). Also, like an increasing number of researchers, he noted the utility of introspective measures in uncovering more information about interaction and argued that research needs to consider and compare positive and negative evidence and implicit and explicit feedback in terms of outcomes. Long made an interesting and potentially controversial point in relation to the durability of any interaction-driven change or learning; he not only pointed out the importance of examining and operationalizing change but also claimed that the importance of demonstrating lasting effects for feedback might not be as important as interaction researchers have assumed, given that short-term change (measured via an immediate posttest, as opposed to via a learner's immediate response during interaction) might be the most valid measure of efficacy.

We see a rather different approach to methodology laid out in the recent work of Swain (2005), whose original formulation of the output hypothesis is often integrated into various models of the interaction-learning relationship as a crucial component of the interaction hypothesis (e.g., Block, 2003). Swain's recent work suggests that there has been a shift of meaning in the output hypothesis, as more attention is paid to the processes of constructing output as opposed to output as a product. Swain has pointed toward a new emphasis in which verbalization contributes to learning through a process that involves reflection and internalization. She argued that this shift in perspective concerning output creates the need for 
new metaphors, new research questions and a new respect for our research tools ... within a sociocultural theory of mind framework, ethnographic and case study approaches would seem to be more valuable at this point in time, although there is certainly a case for more experimental work. (p. 480)

She also pointed out that think-aloud protocols and stimulated recalls might be seen as part of the process of comprehending and reshaping experience that is learning, as opposed to the window into learning that more cognitively oriented approaches often assume. Thus, for Swain, an emphasis on introspection as a learning opportunity as well as a data collection measure is important, as is the collection of in-depth research on individuals within their social contexts.

Gass and Mackey (in press) have also focused on methodological aspects of the interaction model-for example, by pointing to the importance of the need for testing interactionist claims in relation to the role of communicative tasks and interactive settings, the developmental level of learner, the nature of the feedback, and the type of linguistic form. In our recent work (Gass, 2003; Mackey, in press), we have also discussed the importance of understanding the cognitive processes at work in interaction-particularly in relation to issues such as attention and WM-and how investigating these questions will require methodological advances. In particular, we have pointed to the utility of stimulated recall protocols in understanding learners' perceptions about interaction (Gass \& Mackey, 2000). Learners and their perceptions, in terms of how methodologies currently in use might be expanded, are the focus of the next section.

\section{Learners' Perceptions and Introspections}

The current literature provides many examples of interesting methodological choices in regard to the investigation of learners' perceptions about the interaction-learning relationship. A number of researchers have used introspections of some kind to answer a variety of questions concerning, for example, the role of perceptions in learning, how far the perceptions and intentions of learners and instructors (or other interlocutors) overlap, and the role of overlap in noticing and learning (for a recent review, see Egi, 2004). Taking into account Swain's (2005) argument that the introspective techniques used to collect the information about learners' insights might provide learning opportunities, introspection and retrospection seem likely to continue in their growth as methodological tools in interaction research-a trend supported by the fact that incipient research that has made methodological comparisons (Leow \& Morgan-Short, 2004) is generally supportive of their use. At this point, it might be helpful for interaction researchers to consider how introspective techniques can be used more effectively. For example, stimulated recalls are likely to elicit more valuable data if the strength of the stimulus is improved. Videotapes of the learning activity or opportunity (which could show learners carrying out an interactive task) are common but do 
not always catch subtle nonverbal gestures, eye movements, or gaze and, if the interaction is computer based, do not show the keys that are pressed. Adopting techniques commonly used by psycholinguists, such as tracking key or eye movements, might allow interaction researchers to create more effective stimuli; for example, learners might be shown split-screen or picturewithin-picture views where they can see a close-up of their faces and upper bodies as they are interacting, with the other side of the screen or minimized picture showing their computer monitor and the keys they press (and potentially also tracking eye movements during the original recall, stimulated recall, or both). Obviously, detailed training on the part of researchers and subjects is required for such detailed stimuli, together with decisions about when to show these stimuli (concurrently or in response to signals that indicate memory decay on the part of the learner).

A different angle on using introspections and perceptions concerns the investigation of the processes involved in whether and how learners identify interactional feedback as corrective. Experimentally manipulating digitized tapes of interaction, by removing and substituting various features, might also offer a methodological advantage for future researchers to consider as they work to uncover how learners interpret constructs such as recasts, repetitions, and other sorts of response. For example, in an effort to better understand the impact of different features of interaction on learners' interpretations, Carpenter, Jeon, MacGregor, and Mackey (this issue) showed edited clips to learners who commented on them. Taking this sort of experiment one step further, learners could be interviewed about the cues they reported using or not using as they watched tapes. Learners could watch tapes of themselves or of others, with familiar and unfamiliar interlocutors, which involve interactional modifications with forms at different developmental levels. Digital manipulation of interaction, although a step removed in terms of authenticity, holds great promise for providing examples of features that do not occur frequently in naturalistic discourse but that are useful for addressing questions about learning. Researchers in a number of different areas have begun to develop sophisticated tools for tracking computer behavior, and computerassisted language learning research also holds promise in this respect insofar as both manipulation and careful tracking of the features of interaction are methodologically quite feasible.

For interaction researchers, as for SLA in general, forging partnerships with researchers in related areas is likely to result in an expansion of our current tools as well as the questions we can ask. One potentially useful area for collaboration is the rapidly growing area of cognitive neuroscience.

\section{Cognitive Neuroscience}

In the future, the question of how interaction impacts learning might be investigated in collaboration with neuroscientists. Recent methodological advances 
in cognitive neuroscience permit investigation of cognitive processes in real time. Electroencephalography (EEG) and functional magnetic resonance imaging (fMRI) are noninvasive techniques that might prove particularly useful for interaction research. Already used in first language (L1) research and in some second language (L2) research, both techniques provide different but complementary information about how learners process certain types of phonological deviants, semantic incongruities, morphosyntactic violations, and prosodic anomalies in different modalities. EEG-based event-related potentials (ERPs) provide millisecond-by-millisecond information about sentence processing. ERPs index whether and when a participant registers linguistic incongruities. This temporal information can distinguish automatic and controlled processes. fMRI, although not as sensitive to temporal aspects of language as ERPs, provides high-resolution information about the neural regions involved in the processing of a language. In L1 sentence processing, specific regions are differentially activated when participants encounter different types of linguistic input. Different frontal and subcortical regions are associated with WM and morphosyntactic processing, whereas posterior regions are associated with lexicosemantic processes.

In terms of L2s, novice and expert levels of language knowledge might be distinguished in some cases by regional activation differences or patterns. As research in the medical and cognitive psychology literature shows (Ullman, 2005), these techniques can provide useful and interesting information about cognitive functioning. Both ERPs and fMRI might one day be used to advance interaction research. For example, ERPs could be used to investigate learners' responses to recasts in a manner similar to verbal report techniques (stimulated recall, think-aloud protocols, and immediate reports), without incurring interference, degradation, or concerns about reactivity or veridicality. In fact, some ERP research has indicated that low-proficiency learners show altered activation patterns for violations that they are unable to identify on paperand-pencil tests (e.g., Tokowicz \& MacWhinney, 2005). Researchers could use fMRI to investigate activation patterns for regions that include mirror neuronsneurons that fire when someone either performs or watches another person perform an action-as learners experience a recast themselves or hear a recast directed at someone else. Data could reveal (a) whether motor neurons fire during the recast and (b) any relationship between activation intensity and output or learning (which could be measured through pretests and posttests). fMRI could be used for investigating additional and individual variables that might impact SLA and interaction, such as affective state (arousal and depression) and age-related declines.

It is important to note that most research in cognitive neuroscience is in its infancy; moreover, the costs are significant in terms of equipment and training, the time investment involved in researching and developing appropriate stimuli and tasks is considerable, and the potential for misinterpretations and overgeneralizations in relation to such data is high. It will be important to ask carefully constrained questions and make cautious claims in the initial 
stages. Having said this, with their capacity to provide online data related to cognitive representations of SLA and interaction and their broad utility in investigating both language-specific and general learning phenomena, these methodologies hold promise for interaction research if researchers can develop appropriate collaborations with neuroscientists.

\section{Cognitive Psychology and WM Tests}

Psychometric tests of WM capacity, also originating in the cognitive science area, are being more widely used in SLA research in general. Indeed, some SLA researchers have advanced arguments that there is some overlap between measures of WM and measures of language aptitude (Robinson, 2001; Skehan, 1998). In a discussion of two models of WM, N. Ellis (2005) noted that there are two common practices in regard to WM tests in SLA: one that typically involves measuring the phonological loop and one that emphasizes the tradeoff between storage and processing and, thus, measures the sentence span. As Ellis also pointed out, a comprehensive account of different models of WM and associated tests is provided in Miyake and Shah (1999).

A number of SLA researchers have focused on phonological short-term memory (PSTM), one component of what Baddeley (2000) referred to as the working memory system (see also Bunting, Conway, \& Heitz, 2004, for a description of the working memory system). PSTM tests have typically involved the measurement of WM as the ability to repeat novel phonological input (e.g., pseudowords or words in an unknown language) correctly (Ellis \& Schmidt, 1997; Ellis \& Sinclair, 1996). Findings of SLA studies have generally supported a role for PSTM in L2 learning. Likewise, SLA researchers have carried out studies involving sentence span tests (Harrington \& Sawyer, 1992; Walter, 2004) that show a relationship between WM and SLA. Results from research studies to date suggest that WM capacity might be related to the amount and type of benefits that learners garner from oral interaction. For example, it is possible that learners with different WM capacities might benefit differentially from reformulations that provide them with opportunities to make cognitive comparisons without also requiring modifications to their output. WM tests appear to be a promising area for interaction researchers to consider as they attempt to understand individual differences in learning, particularly in relation to variation in the ways learners benefit from interactional feedback.

However, as Juffs (2003) has pointed out, researchers in SLA (and interaction) are not often trained cognitive psychologists and might not be aware of the debates about, for example, exactly what WM tests measure. Also, as N. Ellis (2005) noted, "there has been too little work directly comparing these transatlantic models of working memory and their components as predictors of different aspects of SLA," which points to a danger in "circularity in interpretation and operationalization of the different components" (p. 339). For example, there is a need to better understand the relationship (and contro- 
versy) between what tests of L1 WM and tests of L2 WM measure, or the relationship and significance of different components of the tests, such as sense, grammaticality, and recall, as well as a clear need to understand the relationship among attention, noticing, and WM. Despite these ongoing debates about WM and the methods of testing it, several interesting studies of the role of WM in interaction-driven learning exist, and promising claims and research by Robinson (2003) and Erlam (2005) suggest that, used cautiously, WM testing could add to our understanding of individual differences in interactiondriven learning.

\section{SOCIAL CONTEXT: LEARNERS AND THEIR INTERLOCUTORS}

As noted previously, some researchers have argued for greater recognition of the social context in interaction research (Block, 2003). In a cogent, persuasive, and wittily written analysis of such arguments, Thomas (2005) pointed to some of the problems involved with trying to bridge gaps. Obviously, the term social context encompasses many areas, and it is important to note here, as has been noted elsewhere (Long, 1997), that most interaction researchlike SLA research more broadly_focuses primarily on cognition and learning as opposed to language use. Nevertheless, greater insights into the processes of learning might be provided if interaction researchers began to take up the design challenges involved in incorporating the insights and questions of those who focus on social context. We are beginning to see a move toward this in interaction research-for example, in the work of Swain (2005) as well as the increasing use of language-related episodes (or talk about language; Kowal \& Swain, 1994) as a way to understand interaction data. There is also greater recognition that different kinds of interactional process, and therefore opportunities for learning, might be dependent on different classroom discourse contexts (e.g., Oliver \& Mackey, 2003), and interest in investigations of learner factors, such as literacy in interaction (Bigelow, delMas, Hansen, \& Tarone, in press), is on the rise.

Thus, whereas interaction research, like most of SLA, focuses appropriately (in my view) on development and learning, interaction researchers might benefit from incorporating some understanding of social factors-including the learner, their interlocutor(s), the task, the setting, and so on-into designs that examine learning. In doing so, it is important to keep in mind what Ellis and Barkhuizen (2005) pointed out in response to arguments advocating that research take into account the inherent variability of L2 interactions: "There is no existent methodology for pursuing this reconceptualization" (p. 195). Should questions and methods be brought closer in this respect and, if so, how? For example, one consideration focuses on what learners can bring to interaction, which also relates to the practical applications of the interaction hypothesis. Many of the interaction studies to date assume that learners are passive consumers of the task or the feedback that teachers or interlocutors 
provide (although see Swain, in press). Even though some researchers have suggested that learners' perceptions about the value of interaction might potentially impact the structure of the interaction or whether they benefit developmentally from the interaction (Mackey, in press), most of these sorts of questions have not yet been empirically investigated. It might be worthwhile to ask whether and how we might aid learners to make the most of interaction for themselves and their interlocutors. Methodologically, this could mean creating and manipulating interactive contexts that facilitate learning. For example, raising learners' expectations or sensitivity to interaction as part of an experimental methodology might allow better understanding of learning connections. The L2 writing literature shows that learners can be trained to be peer responders to each other's writing and to use feedback effectively (e.g., Ferris \& Hedgcock, 2005). As N. Ellis (2005) put it, "'notice this,' say our language tutors, in their words and actions, and thus a new wave of explicit analysis is initiated, with consequent benefits from NS-NNS and NNS-NNS oral discussions" (p. 332). Naturally, this approach would need to take into account issues such as explicitness and salience and the fact that, as some researchers would argue, the implicit nature of interactional feedback is one of its key elements.

Experimental interaction research has often used native speaker (NS) interlocutors who feign noncomprehension in the same way that teachers in classrooms do when they want to elicit a particular type of response. In experimental interaction studies, researchers usually aim for these NSs, or teachers, to provide targeted forms, interactional features, or both in a controlled but naturalistic fashion. Sometimes, their input and responses are based on scripts for modified input created by the researcher. Interestingly, McDonough's (this issue) experiment, points to an extension of this sort of manipulation. She engaged the services of a learner's nonnative interlocutor as a confederate of the experimenter. Using learners' peers in this way is a new move in interaction research and could open the door to different sorts of interaction, providing researchers with opportunities to gain insights into various aspects of learners' mental representations and processing. Dörnyei (2002) suggested that the motivation levels of one's conversational partner, as well as oneself, might impact production. This sort of claim might be profitably pursued in interaction research using methods of confederate scripting. Again, a consideration of social factors does not have to mean a switch in emphasis from L2 learning. The operationalization of learning, which is a thorny topic in SLA research, will be discussed next.

\section{OPERATIONALIZING DEVELOPMENT AND CONTINUING QUESTIONS}

The SLA literature is replete with different operationalizations of terms such as acquisition, learning, and development. Production is also studied in terms 
of its potential for learning, leading to more terms such as uptake and modified output. Thus, this issue is not unique to interaction research. Many interaction studies focus narrowly on one or two morphosyntactic forms in order to ensure a careful and cautious approach to learning. However, this leads to questions about whether interaction might also facilitate a more general proficiency or the development of pragmatic knowledge. Criticisms have been made of research that relies solely on learners' responses as opposed to posttests in relation to learning (Long, in press; Mackey \& Philp, 1998). Future interaction research would benefit from considering the learning targets of interaction in terms of different forms, languages, and general proficiencies. We might also want to incorporate into our designs some measure of the impact of interaction on structures for which learners already have explicit knowledge, compared with structures about which learners have less explicit knowledge, as well as the relationship between implicit and explicit knowledge within the interaction approach, taking into account the issue of their control over the forms. Does interactional feedback impact these forms differently? If interaction is more (or less) beneficial when learners have previous knowledge of structures, how much knowledge is necessary? How can posttests be designed to measure these constructs? How can studies be designed that can examine the input, the interaction, and the output and learning over the long term?

More interaction research needs to be designed to take account of learners' different developmental levels. How is developmental level to be measured? Although Pienemann's work (1998) has been used by several interaction studies, many researchers-including those who have used his work-have also pointed to the need for cautious operationalizations and measurements. For example, does interaction work, or work differently, depending on developmental level? The role of interaction in learning also needs to be considered in relation to formulaic language or the learning of constructions as opposed to rule-based exemplars. Because the field of SLA seems increasingly accepting of a role for associative or exemplar learning (e.g., the 2002 special issue of SSLA, "Frequency," which was focused around the work of $\mathrm{N}$. Ellis), it would be helpful for interaction research to operationalize and devise treatments and tests for the impact of interaction on these kinds of phenomena.

\section{CONCLUSION}

As noted in the introduction to this special issue, interaction research has advanced a great deal since its inception almost a quarter of a century ago. The goal of this special issue was to showcase articles that illustrate how the methodological boundaries of interaction research continue to be expanded, with the use of new and interesting methodological angles and techniques to further our insights into the interaction-learning relationship. Although space constraints prevented a lengthy discussion, this epilogue has selectively pointed to a few areas of particular interest for future interaction 
research to consider in creating methodologies to answer questions. As a developing discipline, it will also be necessary for us to continue to be willing and open to new perspectives and to seek them out whenever we can, to keep current with the exciting research being conducted in related fields, and to be active and open about collaborations with researchers in other disciplines. Although still emerging, the interaction research agenda is an exciting one precisely because there are so many open questions and so many potential avenues for investigation as well as possible applications.

\section{REFERENCES}

Baddeley, A. D. (2000). The episodic buffer: A new component of working memory? Trends in Cognitive Sciences, 4, 417-423.

Bigelow, M., delMas, R., Hansen, K., \& Tarone, E. (in press). Literacy and the processing of oral recasts in SLA. TESOL Quarterly.

Block, D. (2003). The social turn in second language acquisition. Washington, DC: Georgetown University Press.

Bunting, M. F., Conway, A. R. A., \& Heitz, R. P. (2004). Individual differences in the fan effect and working memory capacity. Journal of Memory and Language, 51, 604-622.

Dörnyei, Z. (2002). The motivational basis of language learning tasks. In P. Robinson (Ed.), Individual differences and instructed language learning (pp. 137-158). Amsterdam: Benjamins.

Egi, T. (2004). Verbal reports, noticing, and SLA research. Language Awareness, 13, 243-264.

Ellis, N. C. (2005). At the interface: Dynamic interactions of explicit and implicit language knowledge. Studies in Second Language Acquisition, 27, 305-352.

Ellis, N. C., \& Schmidt, R. (1997). Morphology and longer distance dependencies: Laboratory research illuminating the A in SLA. Studies in Second Language Acquisition, 19, 145-171.

Ellis, N. C., \& Sinclair, S. (1996). Working memory in the acquisition of syntax: Putting language in good order. The Quarterly Journal of Experimental Psychology, 49A, 234-250.

Ellis, R., \& Barkhuizen, G. (2005). Analyzing learner language. Oxford: Oxford University Press.

Erlam, R. (2005). Language aptitude and its relationship to instructional effectiveness in second language acquisition. Language Teaching Research, 9, 147-171.

Ferris, D., \& Hedgcock, J. (2005). Teaching ESL composition: Purpose, process, and practice (2nd ed.). Mahwah, NJ: Erlbaum.

Frequency effects in language processing: A review with commentaries [Special issue]. (2002). Studies in Second Language Acquisition, 24.

Gass, S. M. (2003). Input and interaction. In C. J. Doughty \& M. H. Long (Eds.), The handbook of second language acquisition (pp. 224-255). Oxford: Blackwell.

Gass, S. M., \& Mackey, A. (2000). Stimulated recall methodology in second language research. Mahwah, NJ: Erlbaum.

Gass, S. M., \& Mackey, A. (in press). Input, interaction and output in SLA. In B. Van Patten \& J. Williams (Eds.), Theories in SLA. Mahwah, NJ: Erlbaum.

Harrington, M., \& Sawyer, M. (1992). L2 working memory capacity and L2 reading skill. Studies in Second Language Acquisition, 14, 25-38.

Juffs, A. (2003, October). Working memory: Understanding current issues in mainstream psychology. Paper presented at the Second Language Research Forum, University of Arizona, Tucson.

Kowal, M., \& Swain, M. (1994). Using collaborative language production tasks to promote students' language awareness. Language Awareness, 3, 73-93.

Leow, R. P., \& Morgan-Short, K. (2004). To think aloud or not to think aloud: The issue of reactivity in SLA research methodology. Studies in Second Language Acquisition, 26, 35-57.

Lightbown, P. M. (1998). The importance of timing in focus on form. In C. J. Doughty \& J. Williams (Eds.), Focus on form in classroom second language acquisition (pp. 177-196). New York: Cambridge University Press.

Loewen, S., \& Philp, J. (in press). Recasts in the adult L2 classroom: Characteristics, explicitness, and effectiveness. Modern Language Journal.

Long, M. H. (1997). Construct validity in SLA research: A response to Firth and Wagner. Modern Language Journal, 81, 318-23. 
Long, M. H. (in press). Recasts: The story so far. In M. H. Long (Ed.), Problems in SLA. Mahwah, NJ: Erlbaum.

Mackey, A. (in press). Input, interaction and corrective feedback in L2 learning. Oxford: Oxford University Press.

Mackey, A., \& Philp, J. (1998). Conversational interaction and second language development: Recasts, responses, and red herrings? Modern Language Journal, 82, 338-356.

Miyake, A., \& Shah, P. (Eds.). (1999). Models of working memory: Mechanisms of active maintenance and executive control. New York: Cambridge University Press.

Oliver, R., \& Mackey, A. (2003). Interactional context and feedback in child ESL classrooms. Modern Language Journal, 87, 519-543.

Pica, T. (1994). Research on negotiation: What does it reveal about second-language learning conditions, processes, and outcomes? Language Learning, 44, 493-527.

Pienemann, M. (1998). Language processing and second language development: Processability theory. Amsterdam: Benjamins.

Robinson, P. (2001). Individual differences, cognitive abilities, aptitude complexes and learning conditions in second language acquisition. Second Language Research, 17, 368-392.

Robinson, P. (2003). Attention and memory. In C. J. Doughty \& M. H. Long (Eds.), The handbook of second language acquisition (pp. 631-678). Oxford: Blackwell.

Skehan, P. (1998). A cognitive approach to language learning. Oxford: Oxford University Press.

Swain, M. (2005). The output hypothesis: Theory and research. In E. Hinkel (Ed.), Handbook of research in second language teaching and learning (pp. 471-484). Mahwah, NJ: Erlbaum.

Swain, M. (in press). Languaging, agency and collaboration in advanced second language proficiency. In H. Byrnes (Ed.), Advanced language learning: The contributions of Halliday and Vygotsky. London: Continuum.

Tokowicz, N., \& MacWhinney, B. (2005). Implicit and explicit measures of sensitivity to violations in second language grammar: An event-related potential investigation. Studies in Second Language Acquisition, 27, 173-204.

Thomas, M. (2005). Theories of second language acquisition: Three sides, three angles, three points. Second Language Research, 21, 393-414.

Ullman, M. (2005). A cognitive neuroscience perspective on second language acquisition: The declarative/procedural model. In C. Sanz (Ed.), Mind and context in adult second language acquisition (pp. 141-178). Washington, DC: Georgetown University Press.

Walter, C. (2004). Transfer of reading comprehension skills to L2 is linked to mental representations of text and to L2 working memory. Applied Linguistics, 25, 315-339. 